\title{
Nonlinear dynamic analysis of a structure with a friction-based seismic base isolation system
}

\author{
H. M. R. Suy • R. H. B. Fey • F. M. B. Galanti • \\ H. Nijmeijer
}

Received: 26 April 2006 / Accepted: 10 August 2006 / Published online: 20 January 2007

(C) Springer Science + Business Media B.V. 2007

\begin{abstract}
Many dynamical systems are subject to some form of non-smooth or discontinuous nonlinearity. One eminent example of such a nonlinearity is friction. This is caused by the fact that friction always opposes the direction of movement, thus changing sign when the sliding velocity changes sign. In this paper, a structure with friction-based seismic base isolation is regarded. Seismic base isolation can be employed to decouple a superstructure from the potentially hazardous surrounding ground motion. As a result, the seismic resistance of the superstructure can be improved. In this case study, the base isolation system is composed of linear laminated rubber bearings and viscous dampers and nonlinear friction elements. The nonlinear dynamic modelling of the base-isolated structure with the aid of constraint equations, is elaborated. Furthermore, the influence of the dynamic characteristics of the superstructure and the nonlinear modelling of the iso-
\end{abstract}

H. M. R. Suy

NXP Semiconductors Research, High Tech Campus 5, 5656 AE, Eindhoven, The Netherlands

R. H. B. Fey $(\bowtie) \cdot$ H. Nijmeijer

Eindhoven University of Technology, Department of Mechanical Engineering, P.O. Box 513, 5600 MB, Eindhoven, The Netherlands

e-mail: R.H.B.Fey@tue.nl

F. M. B. Galanti

TNO Built Environment and Geosciences, Civil Infrastructure Department, Van Mourik Broekmanweg 6, P.O. Box 49, 2600 AA, Delft, The Netherlands lation system, on the total system's dynamic response, is examined. Hereto, the effects of various modelling approaches are considered. Furthermore, the dynamic performance of the system is studied in both nonlinear transient and steady-state analyses. It is shown that, next to (and in correlation with) transient analyses, steady-state analyses can provide valuable insight in the discontinuous dynamic behaviour of the system. This case study illustrates the importance and development of nonlinear modelling and nonlinear analysis tools for non-smooth dynamical systems.

Keywords Nonlinear analysis - Periodic solutions · Friction · Stick-slip motion - Seismic base isolation

\section{Introduction}

In the past decades, the field of nonlinear dynamics has received a lot of attention. Many scientists have contributed to the development of new theory as well as numerical and experimental methods for modelling and analysis of nonlinear dynamical systems. In recent years, an increasing amount of papers and books have been dedicated to several aspects (e.g. modelling, existence and calculation of solutions, stability, bifurcations) of non-smooth dynamical systems [1-5]. Examples of mechanical systems with non-smooth dynamic behaviour are typically systems with backlash, systems with dry friction elements and systems with contact. The dynamical behaviour of these systems is 
not completely understood yet. Moreover, the numerical analysis of the dynamic behaviour of such systems is often cumbersome, especially for systems with many degrees of freedom. New theory of non-smooth dynamical systems and new numerical methods are thus needed and developed. The subject of this paper is an emerging application of non-smooth dynamics theory in the field of civil engineering. The dynamic behaviour of a system which is protected against seismic excitation via a friction-based base isolation system, will be investigated.

Earthquakes have a large potential for disastrous consequences. Apart from the loss of life, they can cause great economic losses through structural damage. Therefore, in earthquake-prone regions, the seismic resistance of structures is often carefully studied. The conventional design approach of structures in regions where seismicity is insignificant, aims at the design of structural members in such a way that they can withstand all static and dynamic loads elastically. However, in regions where seismic excitation should be taken into account, this design approach might lead to economically unacceptable design solutions, because structural members might become too large. To prevent this, two alternative design concepts can be employed.

In the first alternative design concept, plastic deformation is allowed in special parts of the structure. This strategy is often referred to as the capacity design method $[6,7]$. The parts where plastic deformation occurs (frequently called plastic hinges) are designed for high ductility to ensure global stability of the structure. However, plastic deformation still results in damage of the structure and, possibly, its contents.

In the second alternative design approach, mechanical devices are added to the conventional superstructure to enhance its seismic response. These mechanical devices are part of a system that can be either passive, active or hybrid [7, 8]. Passive systems dissipate (part of) the earthquake energy input and are activated by the earthquake input itself, without the use of an additional power source. Active systems impose forces on the structure to counteract the seismically induced forces. Here, an additional power source is used in combination with a controller to calculate the actuator output. Finally, hybrid systems combine features of both passive and active control systems.

In this study, one special type of passive system is considered, namely friction-based base isolation systems. These systems consist of mechanical devices with non-smooth friction elements that are placed underneath the superstructure to decouple it from the potentially hazardous surrounding ground motion. Although it is well-known that these base isolation systems may exhibit (highly) nonlinear behaviour, they are often modelled linearly in engineering practice, as prescribed in recent building codes [6]. This assumption might lead to an unrealistic representation of the actual dynamic behaviour of the system. With the aid of nonlinear dynamics, models can be developed and analysed that can accurately describe certain phenomena, which, principally, can not be represented by linear models.

Here, in this paper, the influence of the nonlinear modelling of a base isolation system and the dynamic characteristics of a superstructure on the total system's dynamic response, is examined. Furthermore, the dynamic performance of a base-isolated structure is evaluated, based on additional insight in its nonlinear dynamic behaviour, obtained by nonlinear dynamic analyses. These analyses can be divided into transient analyses, in which the response to earthquakes is considered and steady-state analyses, in which periodic excitation is regarded. Although, obviously, the superposition principle does not hold for nonlinear dynamical systems, steady-state analyses may help to detect potential nonlinear resonance frequencies, which may lead to damage of the structure if these frequencies are excited by the earthquake excitation signal.

This paper is organised as follows. In Section 2, a superstructure will be chosen and its dynamic model will be derived with finite element techniques. Furthermore, a modal analysis and dynamic model reduction will be carried out. Next, in Section 3, seismic base isolation will be introduced and a friction-based isolation system, with discontinuous dynamic behaviour, will be chosen. The derivation of the equations of motion of the total base-isolated system will be elucidated in Section 4. With the obtained dynamic model, transient analyses will be considered in Section 5, followed by steady-state analyses in Section 6. Finally, conclusions will be presented in Section 7.

\section{Dynamic modelling of the superstructure}

As a part of the case study, a superstructure has to be chosen. An existing structure is selected, so that the layout and dimensions of the structural elements can easily be adopted. Furthermore, to facilitate the modelling of 

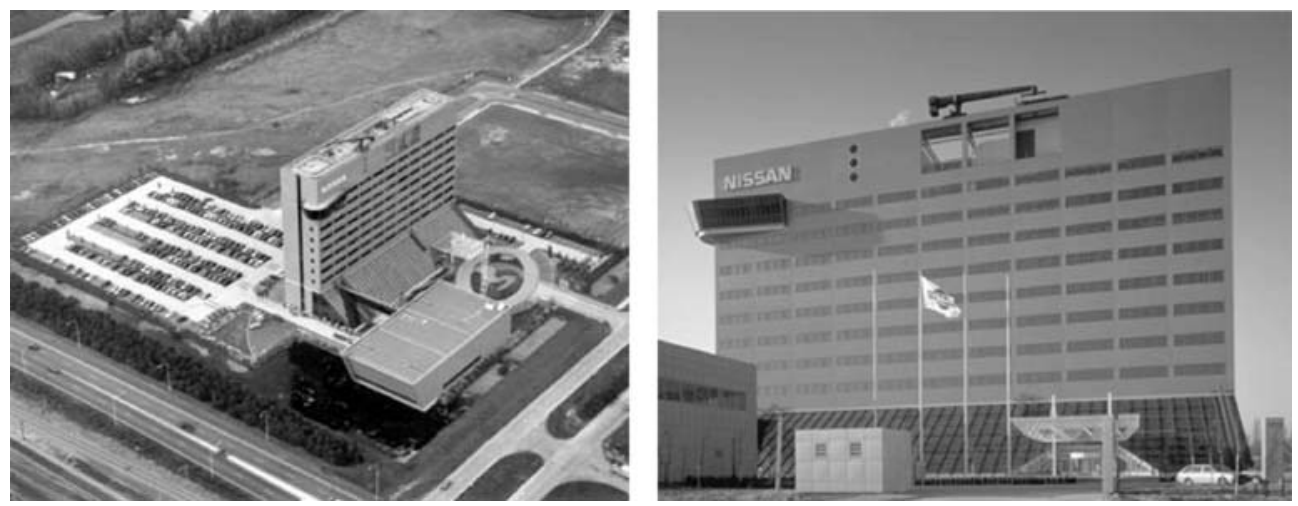

Fig. 1 Overview of the (former) European head office of Nissan, located in Amsterdam

the superstructure, a steel structure without concrete structural members, such as concrete cores and walls, is chosen. Moreover, buildings with steel frames are frequently encountered in regions with large seismic activity, such as Japan and the USA, because of the high ductility and strength of steel. In this study, it is assumed that the structure will always remain in its elastic range (with or without base isolation ${ }^{1}$ ).

The selected superstructure is shown in Fig. 1. This building is the former European head office of Nissan, located in the Netherlands, which has recently been taken over by Mexx International to become their global head office. The fact that this structure is located in a region without seismic activity, is of no importance here, as it is merely used as a part of the case study. It is emphasised that it is our intention to show the influence of nonlinear dynamic modelling and analyses for structures with friction-based base isolation systems, and not to study or improve the seismic resistance of this specific structure. The office is an unbraced highrise steel building of 11 construction levels ( 10 stories) with a total height of $53 \mathrm{~m}$, measured from ground level [9]. It consists of a Moment Resisting Frame without diagonal bracing members and concrete structural members. Each floor surface measures 15 by $80 \mathrm{~m}$ and is formed by concrete floor slabs that do not contribute to the structural stiffness. In addition, the structural stiffness is not significantly influenced by the pre-fabricated concrete wall elements that are attached to the steel frame. Their mass contributions, however, are taken into account in this study.

\footnotetext{
${ }^{1}$ The validity of this assumption is verified in the numerical simulations, as presented later in this paper.
}

Finite element method (FEM) modelling is applied to derive the inertial and stiffness characteristics of this superstructure. The two components of the horizontal ground motion are often not correlated and have their maximum values at different instants. The effect of the vector sum of the two components on the magnitude of the total horizontal motion, can therefore be disregarded [10]. Moreover, rotational excitation can be neglected for a structure of this size and with this symmetric layout [10]. Therefore, only lateral excitation of the building is regarded, as this is the most vulnerable excitation direction of this structure. In this research, both horizontal and vertical excitation will be taken into consideration.

In the FEM-model, each construction level is meshed into $n_{v}$ and $n_{h}$ vertical and horizontal beam elements, respectively. For the FEM-modelling of this frame, Bernoulli-Euler beam elements are employed. Sufficiently accurate results are obtained for meshing parameters $n_{v}=5$ and $n_{h}=4$, as presented in Fig. 2a.

Unlike inertial and stiffness characteristics, it is almost impossible to model the damping of a structure with finite element techniques. Therefore, it is chosen to assign damping ratios to fixed-base eigenmodes of the superstructure. The numerical values of these damping ratios are based on an experimentally determined damping ratio of the fundamental eigenmode of the building under consideration [11] and on the fact that higher modes are more likely to exhibit higher damping, due to larger flexural and shear deformation of the structure [12-14]. The damping ratios of the first four (fixed-base) eigenmodes, as presented in Fig. 2b, are chosen equal to 3, 4, 5 and 6\%, respectively. 


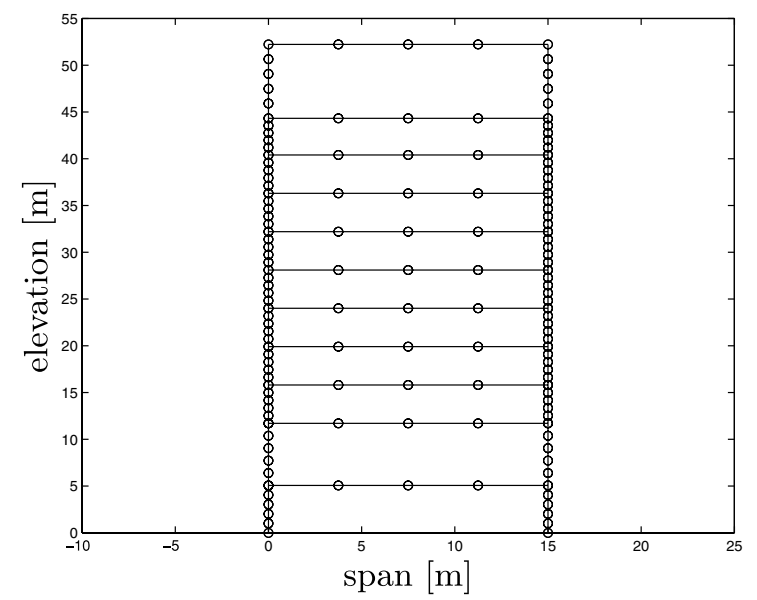

(a)
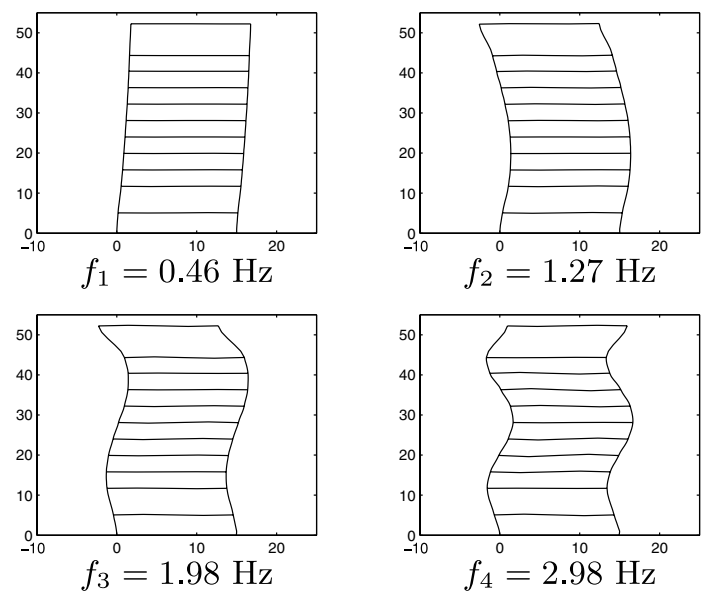

(b)

Fig. 2 FEM-model of the superstructure, with mesh parameters $n_{v}=5$ and $n_{h}=4$ (a). First four eigenmodes and eigenfrequencies of the fixed-base superstructure (b)

With the dynamic model of the superstructure, an exploratory modal analysis is carried out, to evaluate the undamped fixed-base eigenmodes and eigenfrequencies. In the fixed-base situation, the degrees of freedom (dof's) of the two nodes that are located at ground level, are suppressed. In this case, each of these two nodes has three dof's (horizontal and vertical displacement and rotation). These six dof's are called boundary dof's and are, in a later stadium, needed for the coupling of the superstructure to the base isolation system. The remaining dof's of the structure are called internal dof's. The four lowest fixed-base (or fixedinterface) eigenfrequencies equal $0.46,1.27,1.98$ and $2.98 \mathrm{~Hz}$. The corresponding eigenmodes are shown in Fig. 2b.

The meshing of Fig. 2a results in a model with 435 dof's (including the six boundary dof's). Because such a large number of dof's may lead to a computationally inefficient nonlinear dynamic analysis in Sects. 5 and 6 , model reduction is applied. In model reduction, the original set of dof's is transformed into a much smaller set of $n_{g}$ new generalised dof's. In this research, Craig-Bampton reduction [15] is applied to the linear superstructure model. In this method, the displacement field is approximated by a linear combination of static and dynamic modes. The static modes consist of the displacement columns that result when, successively, a unit displacement/rotation is prescribed to one of the boundary dof's, while the other boundary dof's are fixed. These displacement columns are also referred to as constraint modes. The dynamic modes of the Craig-Bampton reduction method consist of the fixed-interface eigenmodes, with eigenfrequencies $f_{i}$ $[\mathrm{Hz}]$, that are within a user-specified frequency band $\left[0<f_{i} \leq f_{\max }\right]$, with cut-off frequency $f_{\max }[\mathrm{Hz}]$. The modes that are outside this frequency band are left out of the transformation matrix. Here, a cut-off frequency of $5 \mathrm{~Hz}$ is taken. This frequency is chosen such that (the largest part of) the dominant frequency range of most earthquakes (between 0.1 and $10 \mathrm{~Hz}[10,16]$ ) is covered. It has been verified with cut-off frequencies of 10 , 15 and $20 \mathrm{~Hz}$ that the use of a larger cut-off frequency does not significantly influence the analysis results, as presented later in the paper, while the number of dof's would be unnecessarily increased (27, 31 and 40 dof's, respectively). This is due to the fact that the majority of the eigenmodes between 5 and $20 \mathrm{~Hz}$ consists of modes with only vertical floor resonance. Compared to lateral eigenmodes (e.g. $f_{1}$ to $f_{4}$ in Fig. 2 b), these vertical modes do not significantly contribute to the structure's seismic response.

Using the Craig-Bampton reduction method with a cut-off frequency of $f_{\max }=5 \mathrm{~Hz}$, the number of dof's of the superstructure is eventually decreased from 435 to 13. It is noted that, in the set of 13 generalised dof's of the reduced model, the set of six boundary dof's is included, to enable coupling of the superstructure to the isolation system. In the remainder of this paper, this reduced superstructure model with $n_{g}=13$ dof's will be used. 


\section{Seismic base isolation}

As mentioned in the introduction, use of a seismic base isolation system may prevent damage to the superstructure in case of an earthquake. In this type of isolation, mechanical devices are placed underneath the superstructure $[7,16,17]$. To ensure that these mechanical devices are deformed uniformly, the superstructure is equipped with a relatively rigid diaphragm at the isolation level. The base isolation system should comply with various characteristics, such as a large degree of lateral flexibility for decoupling of the superstructure from the surrounding ground, vertical load carrying capacity, rigidity to lateral nonseismic service loads and energy dissipation. Therefore, multiple devices are often combined into one system. In this research, a base isolation system is chosen that consists of linear laminated rubber bearings, linear viscous dampers and nonlinear friction elements with rectilinear sliding surfaces [16-18]. In this paper, we will focus on the effect of the latter elements on the system's response.

The laminated rubber bearings consist of alternating layers of natural rubber and steel plates or steel shims that are bonded together. In lateral direction, these isolation devices show a linear behaviour up to moderate shear strains of approximately $100 \%$. The damping of laminated rubber bearings is relatively low (in the order of 3\%) and, therefore, viscous dampers are added. These dampers typically consist of a piston inside an enclosed cylinder, which forces a viscous fluid through or past an orifice. These devices also have a linear characteristic. Finally, the rigidity to nonseismic service loads and the vertical load-carrying capacity of the isolation system are provided by friction elements. These elements consist of two parallel horizontal surfaces, which may slide relative to each other. As friction forces always oppose the relative direction of motion, its behaviour is discontinuous. In literature, various friction models exist that can represent this nonlinear behaviour. In this paper, it is assumed that the friction can be represented by the Coulomb friction model, of which a schematic representation is given in Fig. 3. In this model, the maximum friction force during stiction ( $F_{w, \max }$ in Fig. 3), in which case the relative sliding velocity $(\dot{x})$ is zero, is equal to the friction force during slip $(\dot{x} \neq 0)$. During stiction, the friction force is bounded by $\left[-F_{w, \max }, F_{w, \max }\right]$, counteracting all other external forces, resulting in zero acceleration.

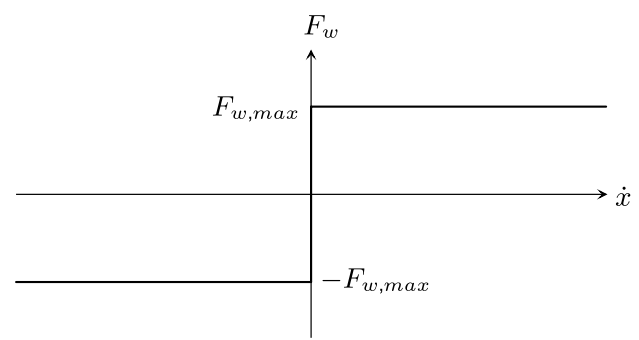

Fig. 3 Schematic representation of the Coulomb friction model

When the external forces exceed the maximum friction force, slip occurs and the friction force equals $\pm F_{w \text {,max }}$ (the sign depending on the sign of $\dot{x}$ ).

\section{Derivation of the equations of motion}

In order to derive the dynamic model of the baseisolated superstructure including Coulomb friction elements, first, the kinematics of the model are elaborated. For this purpose, Fig. 4 is regarded. The two bottom boundary nodes of the superstructure are located at ground level $G L$ and are labelled 1 and 2 . In Fig. 4, the column of dof's of the superstructure, $\underline{q}$, is measured relative to the righthanded coordinate system $(X, Y, Z)$ with origin $O$, which is connected to the surrounding ground. This dof column is given by $\underline{q}=\left[\begin{array}{ll}\underline{q}_{b}^{T} & \underline{q}_{r}^{T}\end{array}\right]^{T}=\left[\begin{array}{llllll}x_{1} y_{1} & \theta_{1} & x_{2} & y_{2} & \theta_{2} & \underline{q}_{r}^{T}\end{array}\right]^{T}$, with $x_{i}, y_{i}$ and $\theta_{i}$ the horizontal, vertical and rotational displacement of boundary nodes $i=1,2$, respectively. Here, $n_{b}$ equals the number of boundary dof's $\underline{q}_{b}$ (six), whereas $\underline{q}_{r}$ denotes the column of $n_{r}$ remaining internal generalised dof's (seven).

The horizontal and vertical ground acceleration, relative to inertial space, and the gravitational excitation $(g)$, are given by the excitation column $\underline{\ddot{u}}=$ $\left[\ddot{u}_{\text {hor }}\left(\ddot{u}_{\text {ver }}+g\right)\right]^{T}$. To each of the boundary nodes 1 and 2 , a base isolation system is attached. This isolation system consists of a linear spring, a linear damper and a nonlinear friction element. The horizontal displacement of the isolation system is also measured relative to $(X, Y, Z)$ and equals $x_{\text {iso }}$. Due to the presence of the relatively rigid diaphragm it holds that: $x_{\text {iso }}=x_{1} \approx x_{2}$. Finally, during numerical simulations, the vertical displacements of the nodes 1 and 2, relative to the surrounding ground, $y_{1}$ and $y_{2}$, are set to zero. The surfaces of the friction elements are thus assumed to remain in contact (with no uplift). The correctness of this 
Fig. 4 Kinematics of the model of the base-isolated structure

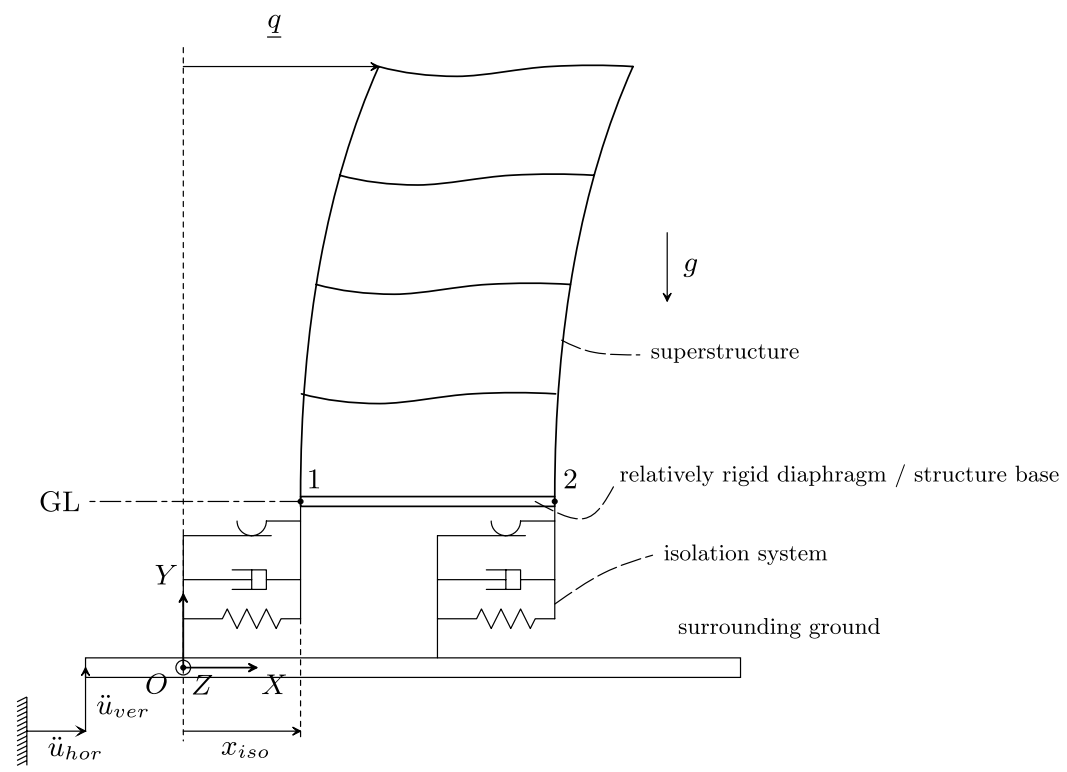

assumption is permanently verified during simulations, as will be explained in Sections 5 and 6.

The dynamic modelling of the linear elements is straightforward and is carried out as follows. At each of the nodes 1 and 2, the combination of one laminated rubber bearing and one viscous damper exerts a horizontal force of

$F_{\text {linear }}=K_{\text {lam }} x_{\text {iso }}+c \dot{x}_{\text {iso }}$.

Herein, $K_{\text {lam }}[\mathrm{N} / \mathrm{m}]$ is the lateral stiffness of a laminated rubber bearing and $c[\mathrm{Ns} / \mathrm{m}]$ denotes the damping constant of this combination. The contribution of the laminated rubber bearings and viscous dampers can be added to the superstructure's (Craig-Bampton reduced) stiffness and damping matrix $K$ and $C$, respectively:

$$
\begin{aligned}
& K^{*} \underline{q}=K \underline{q}+K_{\text {lam }}\left[\begin{array}{lllllll}
x_{\text {iso }} 0 & 0 & x_{\text {iso }} & 0 & 0 & \operatorname{zeros}\left(1, n_{r}\right)
\end{array}\right]^{T}, \\
& C^{*} \underline{\dot{q}}=C \underline{\dot{q}}+c\left[\begin{array}{lllllll}
\dot{x}_{\text {iso }} & 0 & 0 & \dot{x}_{\text {iso }} & 0 & 0 & \operatorname{zeros}\left(1, n_{r}\right)
\end{array}\right]^{T} \text {. (2) }
\end{aligned}
$$

Here, zeros $\left(1, n_{r}\right)$ represents a row of zeros with dimensions $\left(1 \times n_{r}\right)$. Using Lagrange's equations, the following equations of motion of the base-isolated superstructure without friction elements can be derived:

$M \underline{\ddot{q}}+C^{*} \dot{q}+K^{*} \underline{q}=-M \Gamma \underline{\ddot{u}}$.
The (reduced) mass matrix of the superstructure is given by $M$, while $\Gamma$ denotes the excitation influence matrix [10]: $\Gamma=\left[\underline{\Gamma}_{\mathrm{hor}} \underline{\Gamma}_{\mathrm{ver}}\right]$ with $\quad \underline{\Gamma}_{\text {hor }}=\left[\begin{array}{lllllll}1 & 0 & 0 & 1 & 0 & 0 & \Gamma_{r \text { hor }}^{T}\end{array}\right]^{T} \quad$ and $\underline{\Gamma}_{\mathrm{ver}}=\left[\begin{array}{lllllll}0 & 1 & 0 & 0 & 1 & 0 & \Gamma_{r, \mathrm{ver}}^{T}\end{array}\right]^{T} . \quad$ The excitation matrix $\Gamma$ has also undergone the Craig-Bampton reduction from the original set of dof's to the new set of generalised dof's, resulting in the influence columns $\underline{\Gamma}_{r \text {,hor }}$ and $\underline{\Gamma}_{r \text {,ver }}$ for the internal generalised dof's with size $\left(n_{r} \times 1\right)$. Next, the friction elements with Coulomb friction are considered and included in these equations of motion.

A possible mathematical formulation of the Coulomb friction model of Fig. 3 equals [4]:

$F_{w}=F_{w, \max } \operatorname{Sign}(\dot{x})=\mu F_{n} \operatorname{Sign}(\dot{x})$,

where $\mu[-]$ denotes the friction coefficient, $F_{n}[\mathrm{~N}]$ is equal to the normal force between the two friction surfaces, and $\operatorname{Sign}(\dot{x})$ represents the following set-valued function:

$\operatorname{Sign}(\dot{x}) \in \begin{cases}1, & \text { if } \dot{x}>0, \\ -1, & \text { if } \dot{x}<0, \\ {[-1,1],} & \text { if } \dot{x}=0 .\end{cases}$

Consequently, when the relative velocity $\dot{x}$ is zero, the friction force counteracts all other external forces, and is bounded by its range $\left[-F_{w, \max }, F_{w, \max }\right]$. 
To numerically tackle the change of state between stick and slip (or vice versa), a transition phase can be introduced. This so-called switch-model captures the actual non-smooth dynamics of the Coulomb friction model [4]. In the switch-model, the transition state is composed of a narrow band or boundary layer around the hypersurface of the stick phase: $\Omega=$ $\left\{\underline{x} \in \mathcal{R}^{2 n_{g}} \mid \dot{x}_{1}=0 \vee \dot{x}_{2}=0\right\}$, where $\mathcal{R}^{2 n_{g}}$ represents the $2 n_{g}$-dimensional state space $\underline{x}=\left[\begin{array}{ll}q^{T} & \dot{q}^{T}\end{array}\right]^{T}$. The switch band forms a subspace, in which the vector field is such that the solution is forced towards its middle, the hypersurface. The switch band is given by:

$\left|\dot{x}_{i=1,2}\right| \leq \eta$,

with $\eta[\mathrm{m} / \mathrm{s}]$ the width of the switch band. This width has to be chosen small enough to yield a good approximation. Here, a value of $\eta=10^{-5} \mathrm{~m} / \mathrm{s}$ is used. For detailed information about the switch-model, the reader is referred to [4].

Alternatively, the Coulomb friction force can be approximated by the following smooth, but strongly nonlinear model:

$$
F_{w}=\mu F_{n} \frac{2}{\pi} \arctan \left(\epsilon \dot{x}_{\text {iso }}\right) .
$$

The dimensionless parameter $\epsilon$ determines the slope of the function near $\dot{x}_{\text {iso }}=0$. The value of $\epsilon$ should be as large as possible to preserve the non-smooth character of the switch-model (4). However, this will make the equations of motion very stiff, resulting in integration problems. In this study, the value $\epsilon=7500$ appeared to be a good compromise.

After implementation of both the switch-model (4) and the smoothened friction model (7) in the system's equations of motion, only negligible differences were found between the response of the base-isolated building for the two different friction models. This is demonstrated in Fig. 5, where the isolator displacement $x_{\text {iso }}$ is depicted as a function of time. This figure shows the response to a recording of the 1995 Kobe earthquake. More information on transient analyses can be found in Section 5. The derivation of the equations of motion including friction model (7) will be presented now. In this derivation, the method of Lagrangian constraints is used.

The normal forces in the nodes 1 and 2 of Fig. 4 depend on the dynamic response of the superstructure.

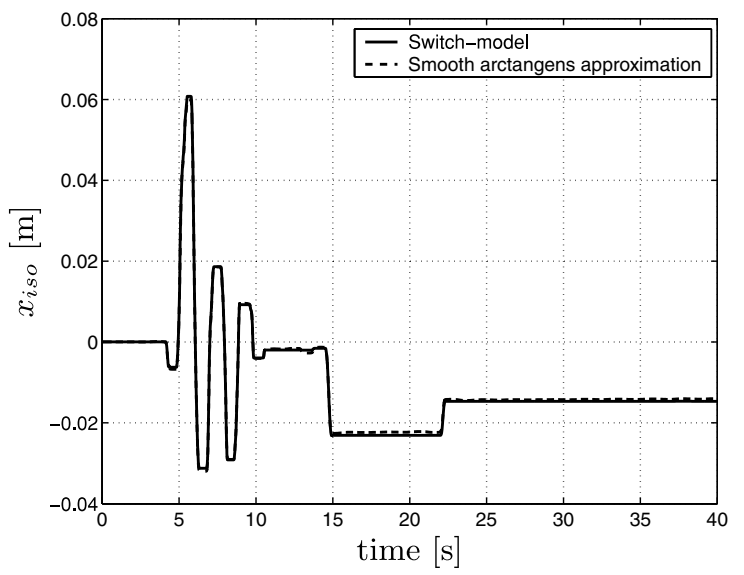

Fig. 5 Isolator displacement $x_{\text {iso }}$ of the base-isolated system as a function of time for a recording of the 1995 Kobe earthquake, for the switch-model (4) and smoothened friction model (7)

These normal forces can be considered as constraint forces, which can be used to check whether the friction surfaces always remain in contact and the vertical displacements of nodes 1 and 2, relative to the surrounding ground, equal zero: $y_{1}=y_{2}=0$. This is the case as long as the normal forces are compressive forces. Because the normal forces are constraint forces, the following formulation is employed, to implement the friction elements in the equations of motion [19]:

$$
\begin{aligned}
M \underline{\ddot{q}}-\underline{h}(\underline{q}, \underline{\dot{q}}, \underline{\ddot{u}})+S \underline{\lambda} & =R^{T} \underline{\lambda}, \\
R \underline{\ddot{q}} & =\underline{0} .
\end{aligned}
$$

These equations can be derived from Lagrange's Equations for Systems with Constraints. In (8a), $\underline{h}(\underline{q}, \underline{\dot{q}}, \underline{\ddot{u}})$ expresses the stiffness and damping characteristics of the superstructure with laminated rubber bearings and viscous dampers and the ground excitation:

$\underline{h}(\underline{q}, \underline{\dot{q}}, \underline{\ddot{i}})=-C^{*} \underline{\dot{q}}-K^{*} \underline{q}-M \Gamma \underline{\ddot{u}}$.

The column $\underline{\lambda}$ denotes the column of constraint forces, which consist of the normal forces in nodes 1 and 2 (see Fig. 4):

$\underline{\lambda}=\left[\begin{array}{l}F_{n_{1}} \\ F_{n_{2}}\end{array}\right]$. 
The constraint forces are introduced in the equations of motion through:

$$
R^{T} \underline{\lambda}=\left[\begin{array}{lllllll}
0 & 1 & 0 & 0 & 0 & 0 & \operatorname{zeros}\left(1, n_{r}\right) \\
0 & 0 & 0 & 0 & 1 & 0 & \operatorname{zeros}\left(1, n_{r}\right)
\end{array}\right]^{T} \underline{\underline{\lambda}},
$$

with zeros $\left(1, n_{r}\right)$ a row of zeros with dimensions $(1 \times$ $n_{r}$ ). The friction forces (which depend on the constraint forces) are introduced via:

$$
S \underline{\lambda}=\left[\begin{array}{cc}
\mu \frac{2}{\pi} \arctan \left(\epsilon \dot{x}_{\text {iso }}\right) & 0 \\
0 & 0 \\
0 & 0 \\
0 & \mu \frac{2}{\pi} \arctan \left(\epsilon \dot{x}_{\text {iso }}\right) \\
0 & 0 \\
0 & 0 \\
\operatorname{zeros}\left(n_{r}, 1\right) & \operatorname{zeros}\left(n_{r}, 1\right)
\end{array}\right]\left[\begin{array}{c}
F_{n_{1}} \\
F_{n_{2}}
\end{array}\right] .
$$

As stated before, the (reduced) column of dof's $\underline{q}$ has been partitioned in the form $\underline{q}=\left[\begin{array}{ll}q_{b}^{T} & \underline{q}_{r}^{T}\end{array}\right]^{T}$, with $n_{b}=6$ boundary dof's $\underline{q}_{b}$ (containing the dof's of nodes 1 and 2) and $n_{r}=7$ internal generalised dof's $\underline{q}_{r}$. Moreover, it is assumed that the coefficient of friction $\mu$ is the same for both friction elements. Finally, it is emphasised that for the switch-model implementation, the expressions for $R, S$ and $\underline{\lambda}$ depend on the system state (stick, slip or transition) and are unequal to the ones mentioned above. For instance, in stick, the friction force is also a constraint force, together with the normal forces.

Equation (8b) represents a formulation of the constraint equation $y_{1}=y_{2}=0$ at acceleration level: $R \ddot{q}=\left[\begin{array}{ll}\ddot{y}_{1} & \ddot{y}_{2}\end{array}\right]^{T}=\underline{0}$. This is done to enable calculation of the unknown accelerations $\underline{\ddot{q}}$ and constraint forces $\underline{\lambda}$. However, this constraint equation is marginally stable with two poles at zero. Small numerical errors may result in $R \ddot{q} \approx \underline{0}$. When this equation is integrated in time, drift of the constraint equation at displacement level may occur. To prevent this, constraint stabilisation is applied [19, 20]. In constraint stabilisation, (8b) is redefined as:

$$
R \underline{\ddot{q}}+\underline{r}_{\text {stab }}=\underline{0},
$$

where:

$\underline{r}_{\text {stab }}=2 \alpha_{\text {stab }} \beta_{\text {stab }} R \underline{\dot{q}}+\alpha_{\text {stab }}^{2} R \underline{q}$,

with initial conditions $R \underline{\dot{q}}_{0}=\underline{0}$ and $R \underline{q}_{0}=\underline{0}$. The parameters $\alpha_{\text {stab }}[1 / \mathrm{s}]$ and $\beta_{\text {stab [-] }}$ are stabilisation parameters. When both parameters are chosen larger than zero, (13) is globally asymptotically stable. Here, $\beta_{\text {stab }}$ is chosen equal to 1 , while $\alpha_{\text {stab }}=2 \pi$, so that (13) is supercritically damped, and the time-steps of the numerical integration are not unnecessarily decreased due to the stabilisation. Combination of (8a) and (13) yields the unknown constraint forces and accelerations:

$$
\begin{aligned}
& \underline{\lambda}=-\left[R M^{-1}\left(R^{T}-S\right)\right]^{-1}\left\{R M^{-1} \underline{h}(\underline{q}, \underline{\dot{q}}, \underline{\ddot{u}})+\underline{\underline{r}}_{\mathrm{stab}}\right\}, \\
& \underline{\ddot{q}}=M^{-1}\left\{\underline{h}(\underline{q}, \underline{\dot{q}}, \underline{\ddot{u}})+\left(R^{T}-S\right) \underline{\lambda}\right\} .
\end{aligned}
$$

With this dynamic model of the base-isolated superstructure, transient and steady-state analyses will be performed in Sections 5 and 6, respectively.

\section{Transient analysis}

In this section, time simulations will be carried out for both the fixed-base and isolated-base structure under earthquake ground motion excitation. This excitation is assumed to act simultaneously at both support points of the system, thus neglecting soil-amplification, soilstructure-interaction and rotational excitation [10].

Before time simulations can be carried out, suitable values of the design variables of the isolation system should be obtained. In this case, the design variables are the lateral stiffness of the laminated rubber bearing $K_{\text {lam }}[\mathrm{N} / \mathrm{m}]$, the damping constant of the combination of the laminated rubber bearing and the viscous damper $c[\mathrm{Ns} / \mathrm{m}]$, and the friction coefficient of the friction elements $\mu$ [-]. Here, it is assumed that both isolation systems at nodes 1 and 2 are identical.

Base isolation design values are found in an iterative line-search, in which two objective functions are minimised, subject to a certain constraint. This so-called parameter line-search is executed for four recordings at different locations of the 1995 Kobe earthquake (all scaled to a horizontal peak ground acceleration (PGA) 
of $\left.3 \mathrm{~m} / \mathrm{s}^{2}\right)^{2}$ to ensure that a broadbanded excitation spectrum is covered [21]. Two seismic performance indicators of structures are chosen as objective functions. These indicators are the interstory drift ratio IDR [-] and the absolute horizontal floor acceleration $a\left[\mathrm{~m} / \mathrm{s}^{2}\right]$. The interstory drift ratio is defined as the quotient of the relative horizontal displacement between two construction levels, and the construction level height. The absolute horizontal floor acceleration, however, is measured relative to inertial space. These two parameters can be related to the amount of (non)structural damage and should, consequently, be minimised [22].

In addition, the isolator displacement amplitude $\bar{x}_{\text {iso }}$ [m] is selected as a constraint function. The isolator displacement may not exceed the seismic gap available between the superstructure's base and the surrounding ground. In our case, the seismic gap is restricted to a maximum width of $400 \mathrm{~mm}$ [7].

The set of design variables which results from the parameter line-search finally equals:

$$
\begin{aligned}
K_{\mathrm{lam}} & =5.0 \times 10^{4} \mathrm{~N} / \mathrm{m}, \\
c & =1.0 \times 10^{4} \mathrm{Ns} / \mathrm{m}, \\
\mu & =0.03 .
\end{aligned}
$$

Subsequently, a comparison is made between the simulation results of the fixed-base situation and the isolated-base situation with design values according to (16). As mentioned before, four recordings (measured at four different seismic stations) of the 1995 Kobe earthquake (all scaled to a horizontal PGA of $3 \mathrm{~m} / \mathrm{s}^{2}$ ) are used as excitation signals. An example of the results for the $I D R$ and $a$ (both defined as the maximum absolute value over the entire time response) as a function of the construction level, is given in Fig. 6a for the Shin-Osaka station record.

Clearly, seismic base isolation greatly benefits the superstructure's seismic performance. This is also demonstrated in Fig. 7a, where the displacement of the top of the building, relative to its base, is given. This displacement is defined as:

$$
\tilde{x}_{\text {top }}=x_{\text {top }}-x_{\text {iso }} \text {, }
$$

\footnotetext{
2 This PGA is chosen such that the fixed-base structure remains in its elastic range for each record. This is done to enable a comparison between the fixed-base and isolated-base situation, later in this section.
}

where $x_{\text {top }}$ and $x_{\text {iso }}$ denote the horizontal displacements of the top of the building and the isolator, relative to the coordinate system $(X, Y, Z)$ of Fig. 4 .

The benefit of base isolation depends on the input excitation spectrum. The benefit of base isolation is, in other words, larger for some excitation records compared to others. This can be observed when Figs. $6 \mathrm{~b}$ and $7 \mathrm{~b}$, which present the results for the KJMA station record, are compared to Figs. 6a and 7a resulting from the Shin-Osaka record. The benefit of isolation is far less for the KJMA input record than for the Shin-Osaka record. With the aid of steady-state nonlinear dynamic analyses, which will be presented in Section 6, this dependency on the input excitation spectrum will be qualitatively clarified.

As mentioned in the introduction, various modelling approximations are used in engineering practice, as prescribed in recent building codes [6]. To study the effect of some of these modelling approaches, earthquake time simulations are again employed. First of all, the effect of taking into account vertical ground excitation is considered. Next, the effect of the varying normal force (constraint force) is investigated. This is done by comparing the results of the original model (where the normal force is calculated as a constraint force to determine the friction force), to the results of a modified model. In this modified model, the normal force in the Coulomb friction model is fixed at its static equivalent, caused by gravitational loading. This implies that, in the case of the modified model, the friction force does not depend on the dynamic response of the superstructure, but is a function of sliding velocity only. Finally, the effect of the dynamics of the superstructure is studied. This is done by comparing the results of the original model with the results of a model where the superstructure is regarded as a rigid body (see Fig. 8).

From these analyses, which will not be discussed in detail here, it can be concluded that the superstructure flexibility greatly affects the total system's response, as it influences the shear forces that are exerted on the isolation system. The other two effects (vertical excitation and varying normal force) have relatively little influence on the response of the isolation system and superstructure.

\section{Periodic solution analysis}

The steady-state behaviour of a periodically excited nonlinear dynamical system is often studied to detect 

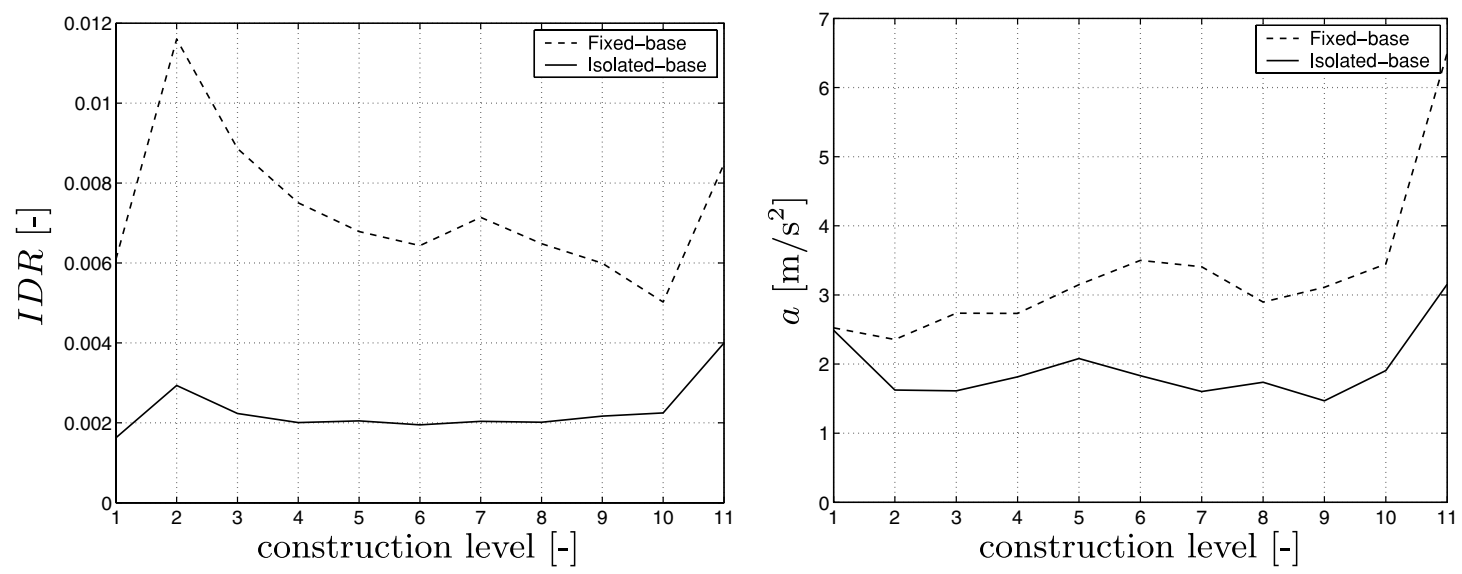

(a)
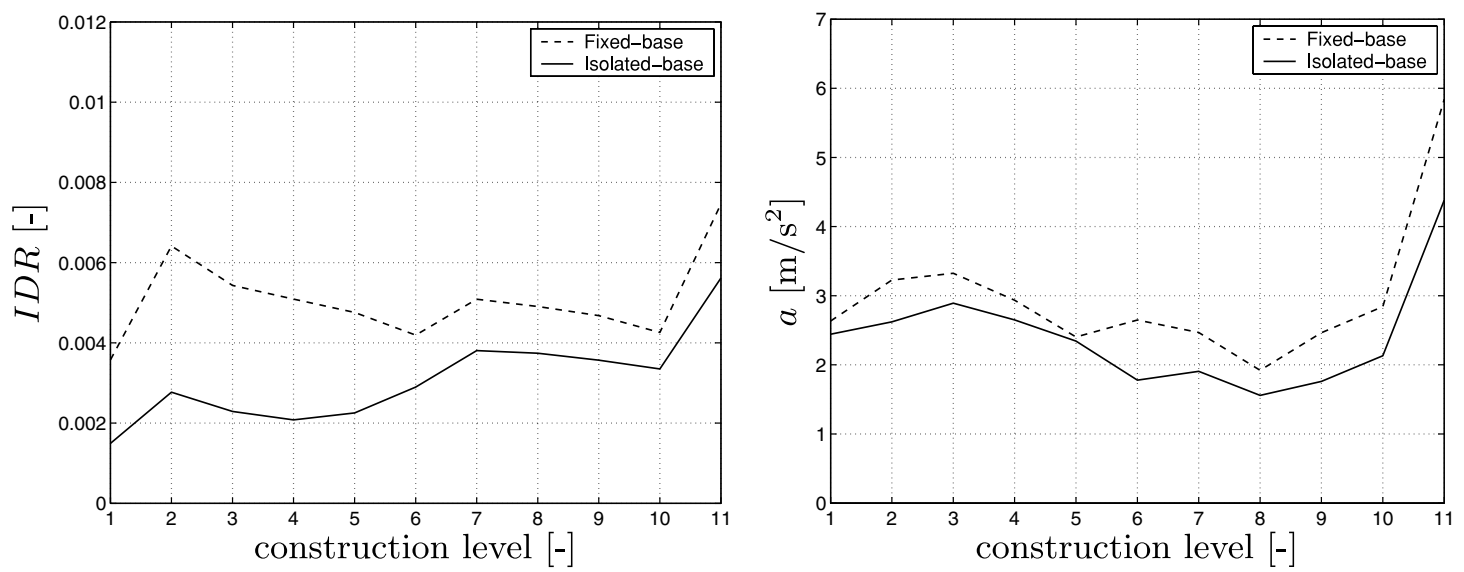

(b)

Fig. 6 Performance indicators $I D R$ and $a$, for the fixed-base and isolated-base system, as a function of the construction level, for the Shin-Osaka (a) and KJMA (b) station record

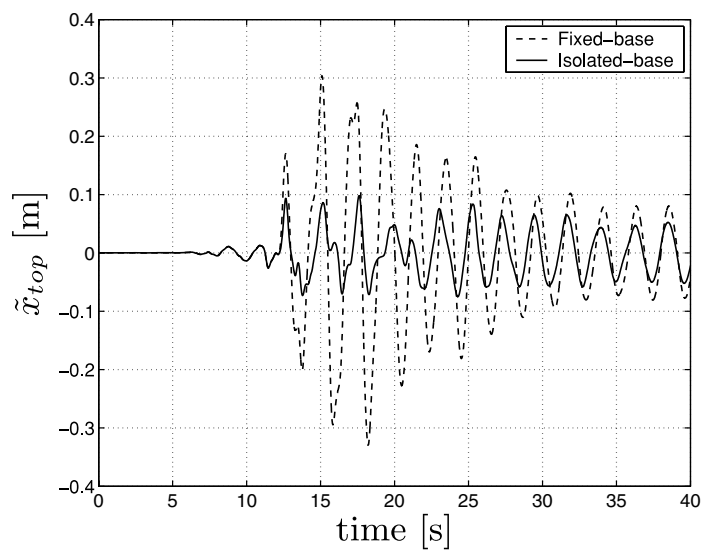

(a)

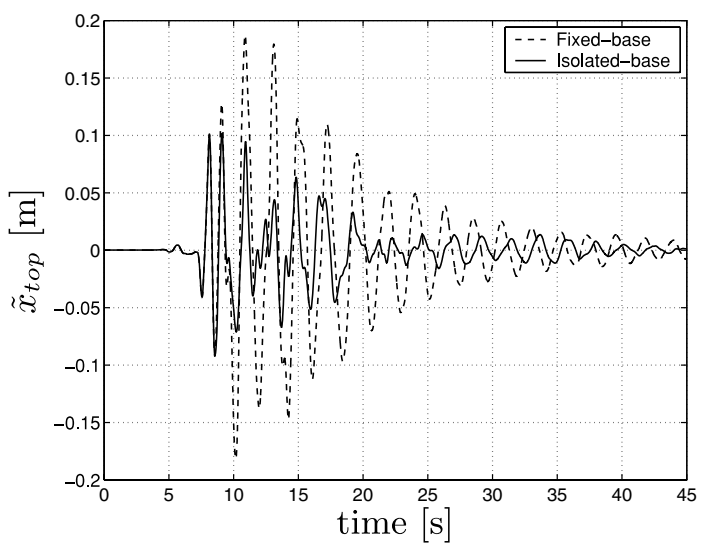

(b)

Fig. 7 Horizontal displacement of the top of the building relative to its base, $\tilde{x}_{\text {top }}$, for the fixed-base and isolated-base system, as a function of time, for the Shin-Osaka (a) and KJMA (b) station record 


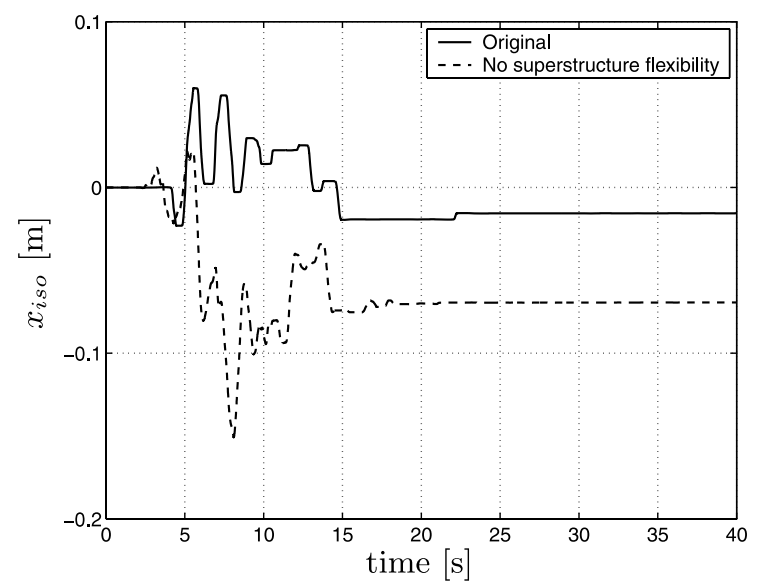

Fig. 8 Isolator displacement $x_{\text {iso }}$ as a function of time for a recording of the 1995 Kobe earthquake, for the case with and without superstructure flexibility

resonance frequencies or bifurcation points which may give rise to undesired dynamic behaviour. The resulting vibrations may have a periodic, quasi-periodic or chaotic nature [23]. Periodic oscillations are characterised by a fixed period time, whereas quasi-periodic solutions can be considered as a countable sum of periodic solutions with incommensurate base-frequencies. A chaotic response exhibits extreme dependence on the initial conditions. In this paper, we focus on finding periodic solutions of the system for excitation frequencies between 0.01 and $10 \mathrm{~Hz}$. The somewhat smaller frequency range of 0.1 to $10 \mathrm{~Hz}$ covers the most important frequencies in an earthquake excitation signal $[10,16]$. Various numerical algorithms exist for efficient calculation of periodic solutions. In this research, the Shooting method is used [23, 24].

Here, for periodic solution analysis, unidirectional excitation is regarded with $\ddot{u}_{\text {ver }}=0$ and:

$\ddot{u}_{\text {hor }}=A_{\text {hor }} \sin \left(2 \pi f_{\text {hor }} t\right)$.

Unidirectional excitation is regarded since the horizontal excitation component of an earthquake signal is usually dominant over the vertical one, and structures are more vulnerable to horizontal ground motion than to vertical ground motion [25]. In (18), $A_{\text {hor }}$ denotes the excitation amplitude $\left[\mathrm{m} / \mathrm{s}^{2}\right]$, while $f_{\text {hor }}[\mathrm{Hz}]$ equals the excitation frequency. In the Shooting method, periodic solutions are found by solving a two-point boundary- value problem. Herein, solutions of the function:

$\underline{H}\left(\underline{x}_{0}, T\right) \equiv \underline{x}_{T}-\underline{x}_{0}=\underline{0}$,

are sought. The column $\underline{x}$ follows from the statespace formulation of the equations of motion (8b): $\underline{x}=\left[\begin{array}{ll}q^{T} & \dot{q}^{T}\end{array}\right]^{T}$. Furthermore, $\underline{x}_{T}$ denotes the solution of the system after a time-lapse $T$, starting from the initial condition $\underline{x}_{0}$ at $t=t_{0}$. The period time of the periodic solution is then given by $T$.

Next, a zero of $\underline{H}\left(\underline{x}_{0}, T\right)$ is (iteratively) found by a Newton-Raphson procedure [23, 24]:

$\frac{\partial \underline{H}}{\partial \underline{x}_{0}} \Delta \underline{x}_{0}=-\underline{H}\left(\underline{x}_{0}, T\right)$.

Evaluation of the Jacobian yields:

$\frac{\partial \underline{H}}{\partial \underline{x}_{0}}=\Phi_{T}\left(\underline{x}_{0}\right)-I$

with $\Phi_{T}$ the monodromy matrix. This matrix can be related to the evolution of solutions of perturbed initial conditions after a period time $T$. The reader is referred to [24] for more information about the monodromy matrix.

Finally, in each iteration $i,(20)$ is solved for $\Delta \underline{x}_{0}$ and the following update is carried out:

$\underline{x}_{0}^{i+1}=\underline{x}_{0}^{i}+\Delta \underline{x}_{0}^{i}$.

During the iterations, the norm of the residual $\epsilon=$ $\left\|\underline{x}_{T}^{i}-\underline{x}_{0}^{i}\right\|$ is evaluated. Convergence of the Shooting method is attained, when the residual reaches a value lower than a certain tolerance $\epsilon_{\text {shoot }}$.

To enable application of the Shooting method to the equations of motion (8), a transformation should be applied from constrained to unconstrained dynamics. Hereto, the following transformation is applied [5]:

$\underline{q}=Q \underline{z}$

with $\underline{z}=\left[\begin{array}{lllll}x_{1} & \theta_{1} & x_{2} & \theta_{2} & \underline{q}_{r}^{T}\end{array}\right]^{T}$ the column of $n_{\min }=11$ independent or minimal dof's, and transformation matrix $Q\left(n_{g} \times n_{\min }\right)$, with $n_{g}$ the number of dof's of generalised dof column $q$. The transformation matrix is chosen such that:

$Q^{T} R^{T}=O$ 
where $O$ equals a zero-matrix with dimensions $n_{\min } \times$ $n_{c}$, with $n_{c}$ the number of constraints (in this case two). With this transformation, the equations of motion (8b) are changed into:

$$
Q^{T} M Q \underline{z}-Q^{T} \underline{h}(Q \underline{z}, Q \underline{\dot{z}}, \underline{\ddot{u}})+Q^{T} S \underline{\lambda}=Q^{T} R^{T} \underline{\lambda}=\underline{0} .
$$

When (8a) and (8b) are combined, the column of constraint forces can be obtained. With (23), this results in:

$$
\underline{\lambda}=-\left[R M^{-1}\left(R^{T}-S\right)\right]^{-1} R M^{-1} \underline{h}(Q \underline{z}, Q \underline{\dot{z}}, \underline{u}) .
$$

It is noted that, due to the transformation, constraint stabilisation (13) and (14) has become obsolete. By combining (25) and (26), the column of accelerations of the minimal dof's then equals:

$$
\begin{aligned}
\ddot{z}= & \bar{M}^{-1} Q^{T}\left\{I+S\left[R M^{-1}\left(R^{T}-S\right)\right]^{-1} R M^{-1}\right\} \\
& \times \underline{h}(Q \underline{z}, Q \underline{\dot{z}}, \underline{\ddot{u}}),
\end{aligned}
$$

with $\bar{M}=Q^{T} M Q$. By numerical integration of (27), the value of $\underline{x}$ (which has now become $\underline{x}=\left[\begin{array}{ll}z^{T} & \dot{z}^{T}\end{array}\right]^{T}$ ) after a time-lapse $T, x_{T}$, can be evaluated and a periodic solution can be found with the Shooting method.

With the aid of path following techniques, periodic solutions can be tracked for a changing system parameter, yielding a branch of periodic solutions. Here, sequential continuation is used to calculate the periodic solutions as a function of the horizontal excitation frequency $f_{\text {hor }}$. In sequential continuation, the periodic solution at some value $f_{\text {hor }}$ is used to find a periodic solution at $f_{\text {hor }}^{*}=f_{\text {hor }}+\Delta f$ with the aid of the Shooting method, which is likely to converge for small $\Delta f$. However, in this research, in some frequency regions the convergence of the Shooting method is poor. In these regions stepped frequency sweeping based on standard numerical integration is applied. The length of this time simulation is chosen long enough to ensure that transients have died out and a steady-state solution is reached. A disadvantage of this method is that it is a relatively time-consuming procedure.

The results of the sequential continuation are given in Fig. 9. Herein, the interstory drift ratio $I D R$, absolute horizontal floor acceleration $a$ (both defined as the maximum value over the entire excitation period and all construction levels), and isolator displacement am-
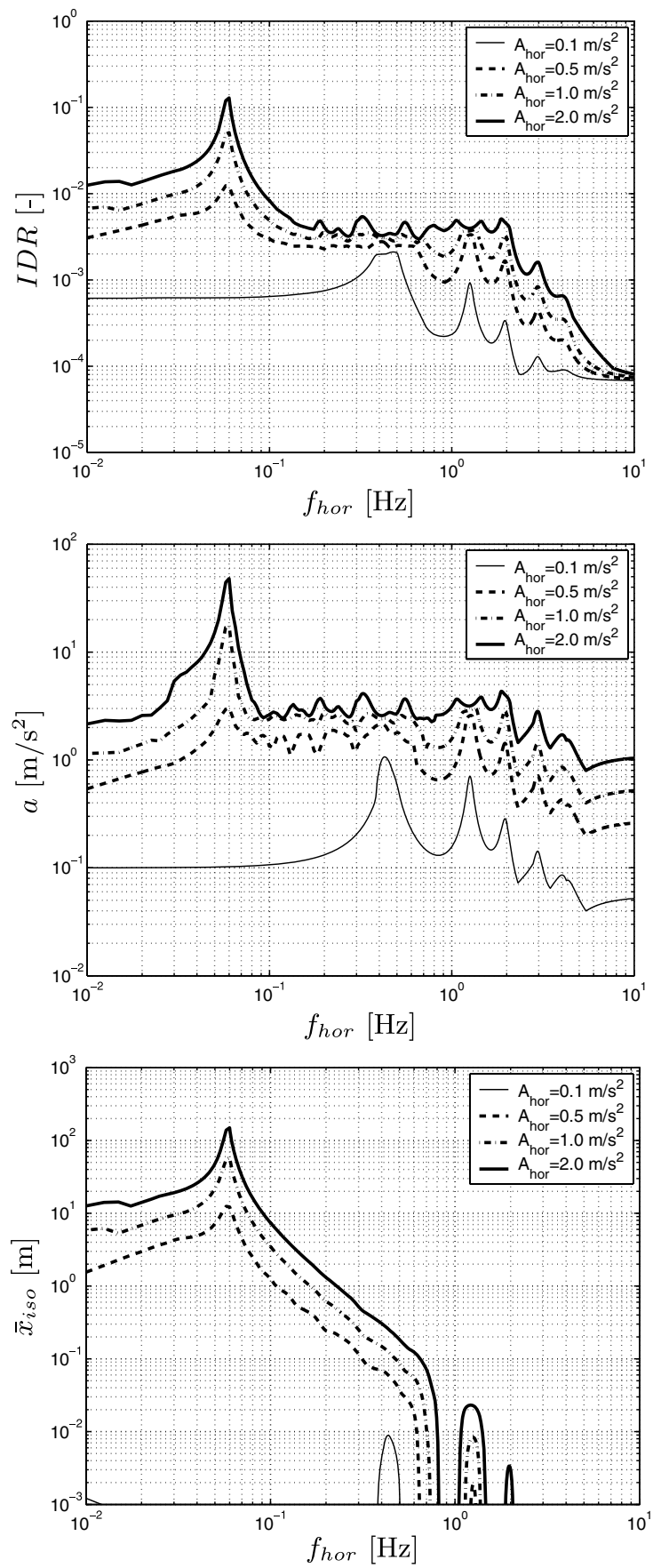

Fig. 9 Frequency amplitude diagram of $I D R, a$ and $\bar{x}_{\text {iso, }}$, as a function of $f_{\text {hor }}$, for various excitation amplitudes $A_{\text {hor }}$, with $K_{\text {lam }}=5.0 \times 10^{4} \mathrm{~N} / \mathrm{m}, c=1.0 \times 10^{4} \mathrm{Ns} / \mathrm{m}$, and $\mu=0.03$

plitude $\bar{x}_{\text {iso }}$, are shown as a function of $f_{\text {hor }}$ for various acceleration excitation amplitudes $A_{\text {hor }}$. These excitation amplitudes are based on the magnitudes of order of the PGA of most earthquakes [21]. 
In the lower graph of Fig. 9, a distinction can be made between frequency regions where $\bar{x}_{\text {iso }}=0$ (consequently, the isolator displacement amplitude is not indicated in the double-logarithmic graph) and frequency regions where the isolator exhibits stick-slip motion with $\bar{x}_{\text {iso }} \neq 0$. In the frequency range above 2.5 $\mathrm{Hz}$, for all values of $A_{\text {hor }}$ considered, the system is in stick during the entire period. ${ }^{3}$ Hence, in this frequency range, the system can be regarded as a fixed-base superstructure and fixed-base eigenfrequencies can be distinguished in the results for $I D R$ and $a$.

When the excitation amplitude is increased, the shear forces that are exerted by the superstructure on the isolation system, are increased as well. Therefore, the larger the excitation amplitude, the larger the frequency regions with isolator stick-slip motion. In addition, the shear forces that are exerted on the isolation system are the largest near the resonance frequencies of the superstructure. This is the reason why in Fig. 9, for example, for a value of $A_{\text {hor }}=2.0 \mathrm{~m} / \mathrm{s}^{2}$, isolator stick-slip motion is induced around the second and third fixed-base eigenfrequency at 1.27 and $1.98 \mathrm{~Hz}$, respectively (see also Fig. 2b). Finally, for very low excitation amplitudes (e.g. $A_{\text {hor }}=0.1 \mathrm{~m} / \mathrm{s}^{2}$ ), the system acts as a fixed-base superstructure over almost the entire frequency range, except for a small frequency band near the first fixed-base eigenfrequency at $0.46 \mathrm{~Hz}$.

In Fig. 9, a harmonic resonance frequency can be distinguished at approximately $0.06 \mathrm{~Hz}$. This base isolation resonance frequency $f_{\text {bis }}$ can be approximated by the eigenfrequency of a single-degree of freedom system, where the superstructure is considered to be a rigid body with mass $M_{s}$, supported by an isolation system without friction elements:

$$
f_{\text {bis }}=\frac{1}{2 \pi} \sqrt{\frac{2 K_{\text {lam }}}{M_{s}}} .
$$

This is caused by the fact that, near the base isolation resonance frequency, stiction is relatively insignificant compared to slip. To decrease structural damage due to earthquakes, the base isolation system is designed such that this base isolation resonance frequency is be-

\footnotetext{
${ }^{3}$ Note that actual stiction is only approximated by the smooth arctangens formulation of the Coulomb friction model. However, these results are all verified with the switch-model with stiction.
}

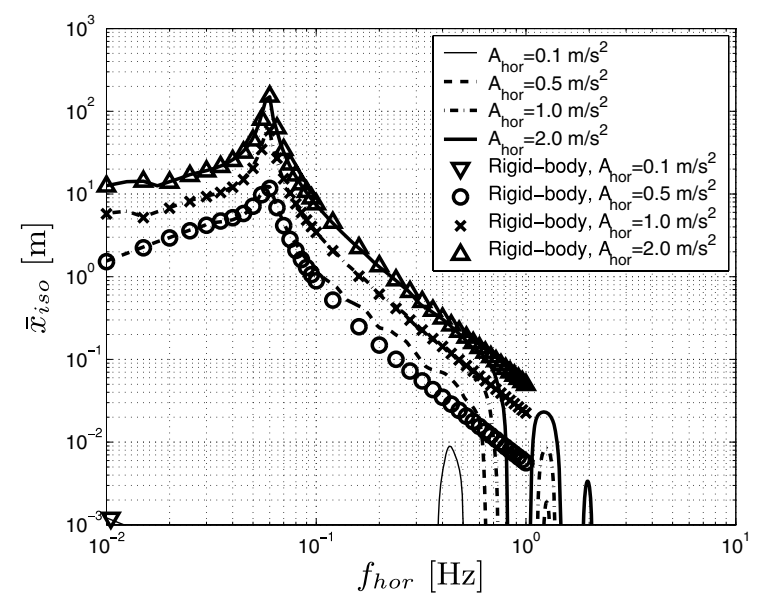

Fig. 10 Frequency amplitude diagram of $\bar{x}_{\text {iso }}$, as a function of $f_{\text {hor }}$, compared to the results of a model with rigid-body superstructure

low the most dominant excitation frequency range of earthquakes $(0.1$ to $10 \mathrm{~Hz})$. In addition, in the low frequency range up to about $0.5 \mathrm{~Hz}$, the superstructure can be regarded as a rigid body. This is demonstrated in Fig. 10, where the results of the original model are compared to the results of a single-dof model with rigid body superstructure, supported by the isolation system with friction elements. This resemblance increases with increasing excitation amplitude.

The excitation is chosen such that the acceleration amplitude $\left(A_{\text {hor }}\right)$ is the same for all frequencies. For very low frequencies, this implies that large excitation displacements result. These large displacements (and the resulting isolator displacements) are physically unrealistic. A more realistic excitation signal is typically based on a constant excitation displacement amplitude in the low frequency range, a constant excitation velocity amplitude in the middle frequency range, and a constant excitation acceleration amplitude in the high frequency range [10]. However, the objective of this paper is merely to show that steady-state analyses may give valuable additional insight in the nonlinear dynamic behaviour of a friction-based base-isolated superstructure, next to the standard transient analyses. Here, several acceleration amplitude levels are employed to study the effect of the excitation amplitude on the dynamic behaviour of the system.

With the aid of the frequency amplitude diagrams, also a comparison can be made between the steady-state periodic behaviour of the superstructure in the fixedbase and isolated-base situation. This is demonstrated 

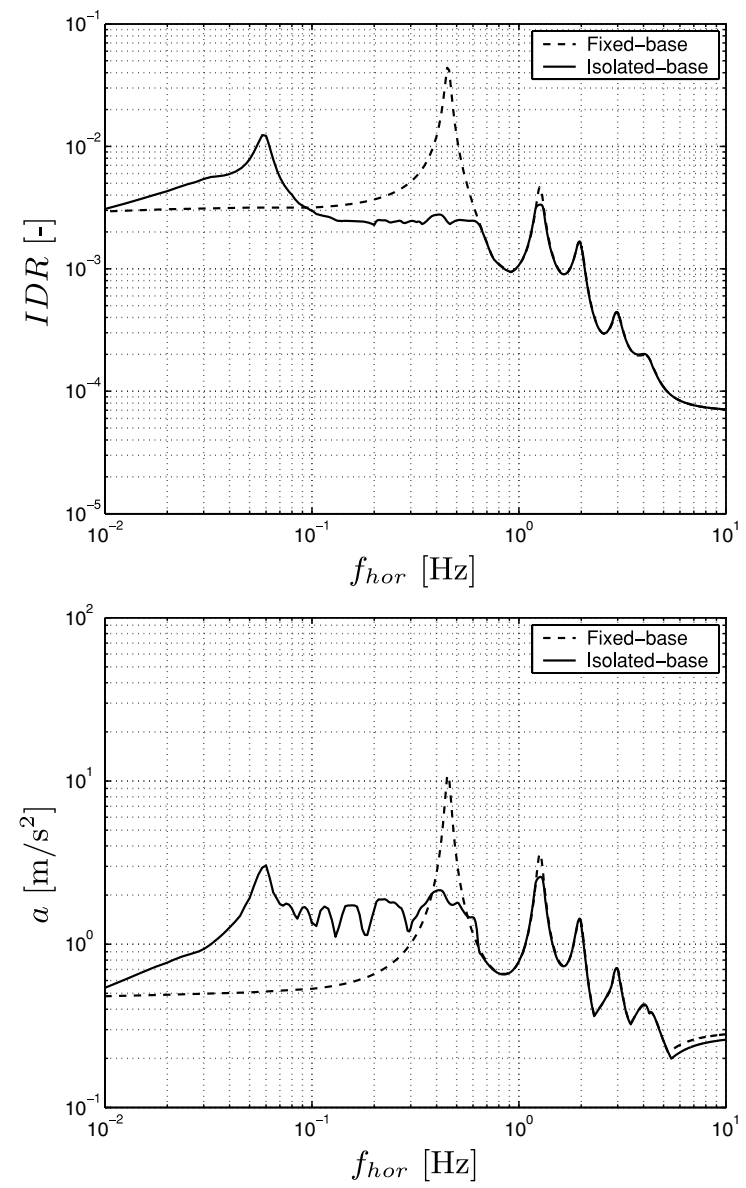

Fig. 11 Frequency amplitude diagram of $I D R$ and $a$ in the fixed-base and isolated-base situation, as a function of $f_{\text {hor }}$, for an excitation amplitude of $0.5 \mathrm{~m} / \mathrm{s}^{2}$

in Fig. 11 for an acceleration excitation amplitude of $0.5 \mathrm{~m} / \mathrm{s}^{2}$. In the fixed-base situation, the system is linear and, therefore, the frequency amplitude diagrams can be regarded as (linear) frequency response functions (FRF's). From Fig. 11, it can be seen that the seismic resistance is improved in the frequency range of 0.1 to $1 \mathrm{~Hz}$. Levels of IDR and $a$ are largely decreased near the first fixed-base eigenfrequency at 0.46 $\mathrm{Hz}$. For higher frequencies, the system's dynamic behaviour is not affected by base-isolation, because the isolation system is in stick during the entire period. The improvement between 0.1 and $1 \mathrm{~Hz}$, however, is at the expense of a deteriorated performance around the base isolation resonance frequency (in this case approximately $0.06 \mathrm{~Hz}$ ). In general, though, the acceleration excitation amplitudes in the frequency range up to 0.1 $\mathrm{Hz}$ are small (earthquakes are most dominant between
0.1 and $10 \mathrm{~Hz}[10,16])$. Indeed, recall that this is the reason for designing the base isolation system such that the base isolation resonance frequency (28) is below $0.1 \mathrm{~Hz}$. Hence, a base isolation system generally benefits the transient response of structures to earthquake excitation.

In addition, these nonlinear frequency amplitude diagrams have been used to study the effect of all three isolator design variables $\left(K_{\mathrm{lam}}, c\right.$ and $\mu$ ) on the seismic isolation characteristics. From these parameter studies, it is concluded that for increasing friction coefficient $\mu$, the frequency ranges where the isolator remains in stick during the entire period, are increased as well. Obviously, in the frequency ranges where the isolator remains in stick, the isolator stiffness and damping constant have no influence. These two design variables do influence the base isolation resonance frequency, which can be approximated by (28), and the corresponding amplitude of this resonance.

Finally, the steady-state analyses are qualitatively correlated with the transient analyses of Section 5. From the frequency amplitude diagrams in Fig. 9, it can be stated that isolator slip mainly occurs in the low frequency excitation range (up to $1 \mathrm{~Hz}$ ). Therefore, earthquake input spectra with a relatively large energy content in this frequency range, will result in a larger isolator displacement. This also provides some indication about the level of uncoupling of the superstructure from the ground motion. In general, the larger the isolator displacement, the larger the degree of uncoupling and the smaller the amount of seismic energy that is transferred to the superstructure. This clarifies why, as mentioned in Section 5, the extent of benefit of base isolation depends on the input excitation spectrum. The power spectral density (PSD) of the four different excitation records of Section 5 can be used to elucidate why the largest benefit of base isolation is obtained for the Shin-Osaka record. This record is namely relatively dominant in the low frequency range between 0.2 and $0.8 \mathrm{~Hz}$, compared to the KJMA record and the two other records.

Summarizing, the steady-state analyses seem to provide a link between the PSD of the earthquake excitation signals and the extent of the benefit of isolation. Still, the nonstationarity of these excitation signals and the fact that modal superposition can not be applied to nonlinear systems, make a clear interpretation of this link very difficult. 


\section{Conclusions}

In this paper, the modelling and analysis of a nonsmooth base-isolated structure has been discussed. This study forms an example of an emerging application of the modelling and analysis of non-smooth dynamics in the field of civil engineering. The base isolation system, consisting of a combination of laminated rubber bearings, viscous dampers and friction elements, has been chosen to seismically isolate a building of 11 construction levels. A dynamic model of this building has been derived with finite element techniques, after which dynamic model reduction has been performed to reduce the number of degrees of freedom. A smooth approximation of the discontinuous behaviour of friction has been used in the derivation of the equations of motion of the base-isolated structure, with the aid of Lagrange's method for systems with constraints. The results based on this modelling approach of the friction elements, have been verified with the results based on a non-smooth switch-band model.

In transient time simulations, the benefit of base isolation has been demonstrated for recordings of the Kobe earthquake. The extent of benefit of base isolation depends on the input excitation spectrum. In addition, the influence of various modelling approaches has been studied. It can be concluded that the dynamics of the superstructure (superstructure flexibility) largely influence the total system's dynamic response. Other effects, such as the vertical ground acceleration and the varying normal force on the friction elements, have relatively little influence on the isolator displacement and the superstructure response.

Steady-state analysis with the aid of periodic solution solvers provides valuable insight in the nonlinear dynamic behaviour of the base-isolated structure. A base isolation resonance frequency has been distinguished in the results, which can be approximated by the eigenfrequency of a single-degree of freedom system, where the superstructure is regarded as a rigid body, supported by the isolation system without friction elements. The obtained frequency amplitude diagrams have revealed that, for the acceleration excitation levels considered, the friction-based base isolation system is only activated in the low frequency excitation range up to $1 \mathrm{~Hz}$. For higher frequencies, the isolation system remains in stick during the entire period of the periodic solution and the system acts as a fixed-base structure. In conclusion, it is stated that the steady-state analy- ses of this non-smooth dynamical system can be used to (qualitatively) clarify the dependency of the benefit of base isolation on the input excitation spectrum. Therefore, it may be expected that, in the future, theory and numerical tools for non-smooth dynamical systems may prove to be very useful in the design of earthquake resistant structures.

Acknowledgements This research was partially supported by the EU Network of Excellence HYCON (contract number FP6IST-511368). This work is based on the first author's M.Sc. project at the Department of Mechanical Engineering of the Eindhoven University of Technology, Eindhoven, The Netherlands.

\section{References}

1. Filippov, A.F.: Differential Equations with Discontinuous Right-Hand Sides. Kluwer Academic, Dordrecht (1988)

2. Brogliato, B.: Nonsmooth Mechanics, 2nd edn. Springer, London (1999)

3. Yakubovich, V.A., Leonov, G.A., Gelig, A.Kh.: Stability of Stationary Sets in Control Systems with Discontinuous Nonlinearities. Series on Stability, Vibration and Control of Systems, Series A, vol. 14, World Scientific, New Jersey (2004)

4. Leine, R.I., Nijmeijer, H.: Dynamics and Bifurcations of Non-Smooth Mechanical Systems. Lecture Notes in Applied and Computational Mechanics, vol. 18, Springer, Berlin (2004)

5. Glocker, C.: Set-Valued Force Laws: Dynamics of NonSmooth Systems. Lecture Notes in Applied and Computational Mechanics, vol. 1, Springer, Berlin (2001)

6. ECS: EN 1988 Eurocode 8: Design of Structures for Earthquake Resistance, Part 1: General Rules, Seismic Actions and Rules for Buildings (draft number 4, ref. no. prEn 19881:200X), European Committee for Standardization (2001)

7. Oliveto, G. (ed.): Innovative Approaches to Earthquake Engineering. WIT Press, Southampton (2002)

8. Casciati, F.: Structural control for dynamic hazard mitigation, In: Advanced Dynamics and Control of Structures and Machines. Irschik, H., Schlacher, K. (eds.) Springer,Verlag (2004)

9. Kraus, J., Derkink, M.: Headquarters Nissan Europe in Amsterdam. Bouwen met Staal, 23(3). In Dutch (1990)

10. Clough, R.W., Penzien, J.: Dynamics of Structures, 2nd edn. McGraw-Hill, New York (1993)

11. van Duin, F.: Dempingsmetingen in vier gebouwen die in hoogte variëren tussen 22 en 54 meter (technical report), TNO Building and Construction Research, Delft, report number 2000-CON-DYN-R2058. In Dutch (2000)

12. Chopra, A.: Dynamics of Structures: Theory and Application to Earthquake Engineering, 2nd edn. Prentice-Hall, Upper Saddle River (2001)

13. Paz, M.: Structural Dynamics: Theory and Computation, 4th edn. Springer-Verlag, New York (1989) 
14. Satake, N., Suda, K., Arakawa, T., Sasaki, A., Tamura, Y.: Damping evaluation using full-scale data of buildings in Japan. J. Struct. Eng. 129(4), 470-477 (2003)

15. Craig, R.R. Jr.: Coupling of substructures for dynamic analyses: an overview. In: 41st AIAA/ASME/ASCE/AHS/ASC Structures, Structural Dynamics, and Materials Conference; AIAA-2000-1573, Atlanta, GA, April 3-6 (2000)

16. Skinner, R., Robinson, W., McVerry, G.: An Introduction to Seismic Isolation. Wiley, Chichester (1993)

17. Naeim, F., Kelly, J.: Design of Seismic Isolated Structures: From Theory to Practice. Wiley, New York (1999)

18. Komodromos, P.: Seismic Isolation for Earthquake Resistant Structures. WIT Press, Southampton (2000)

19. Haug, E.: Computer Aided Kinematics and Dynamics of Mechanical Systems, Volume I: Basic Methods. Allyn and Bacon, Boston (1989)
20. Baumgarte, J.: Stabilization of constraints and integrals of motion in dynamical systems. Comput. Methods Appl. Mech. Eng. 1, 1-16 (1972)

21. Pacific Earthquake Engineering Research Center (PEER) (2004) Strong motion database. Retrieved from http://peer.berkeley.edu/smcat. cited 8 June (2004)

22. Porter, K., Kiremidjian, A., Legure, J., King, S.: A building damage estimation method for business recovery. In: 12th World Conference on Earthquake Engineering, Auckland, New Zealand, January 30-February 6 (2000)

23. Parker, T.S., Chua, L.O.: Practical Numerical Algorithms for Chaotic Systems. Springer-Verlag, New York (1989)

24. Leine, R.I., van Campen, D.H., de Kraker, A., Van den Steen, L.: Stick-slip vibrations induced by alternate friction models. Nonlinear Dyn. 16, 41-54 (1998)

25. Japanese Society of Civil Engineers: Dynamic Analysis and Earthquake Resistant Design: Strong Motion and Dynamic Properties, vol. 1, Balkema, Rotterdam (1997) 